\title{
Strength and elasticity of niobium under high pressure
}

\author{
Anil K. Singh ${ }^{1, a)}$ and Hanns-Peter Liermann ${ }^{2}$ \\ ${ }^{1}$ Materials Science Division, National Aerospace Laboratories, Council of Scientific and Industrial Research \\ (CSIR), Bangalore 560 017, India \\ ${ }^{2}$ Petra III, P02, Deutsches Elektronen Synchrotron, Notkestr. 85, 22607, Hamburg, Germany
}

(Received 22 December 2010; accepted 26 April 2011; published online 15 June 2011)

\begin{abstract}
High purity polycrystalline niobium contained in boron-epoxy gasket was compressed in a diamond anvil cell (DAC). The pressure was increased in steps of $\sim 3 \mathrm{GPa}$ and the diffraction patterns recorded at each pressure with the incident $\mathrm{x}$-ray beam perpendicular to the load axis of the DAC (radial diffraction). The maximum pressure reached was $37.6 \mathrm{GPa}$. The compressive strength (differential stress) derived from the radial diffraction data is $0.44(1) \mathrm{GPa}$ at $2.1 \mathrm{GPa}$ and shows a shallow maximum at $\sim 5 \mathrm{GPa}$, and then decreases to $0.35(5)$ at $12 \mathrm{GPa}$. At higher pressures, strength increases nearly linearly and the extrapolated value at $40 \mathrm{GPa}$ is $0.94(6) \mathrm{GPa}$. At any pressure, the single-crystal elastic moduli derived from the diffraction data can be made to match well those obtained from the extrapolation of the elasticity data at ambient pressure by adjusting the weight parameter $\alpha$ that appears in the lattice strain theory. The parameter $\alpha$ is found to decrease from 2.00(8) at $2.1 \mathrm{GPa}$ to $1.35(4)$ at $37.6 \mathrm{GPa}$. (C) 2011 American Institute of Physics. [doi:10.1063/1.3594748]
\end{abstract}

\section{INTRODUCTION}

Niobium, a $4 d$ transition metal with a body-centered cubic (bcc) structure, has been studied extensively under pressure. The pressure-volume relation has been measured by many investigators under static pressures by $\mathrm{x}$-ray diffraction technique $^{1-5}$ and under shock loading. ${ }^{6}$ The stability of the bcc phase at high pressures investigated by first-principles total energy calculations suggests a transformation of the bcc phase to a face centered cubic structure at very high compression. ${ }^{7}$ The measurement of the superconducting transition temperature $T_{c}$ as a function of pressure indicates anomalies at 5 and $60 \mathrm{GPa}$ in the $\mathrm{T}_{\mathrm{c}}$-pressure plot. ${ }^{8}$ These anomalies have been attributed to electronic topological transitions (ETTs). ${ }^{9}$ The volume effect associated with ETT is of general interest. High-precision measurement of equation of state suggests that the volume effect, if any, associated with the ETT in $\mathrm{Nb}$ is extremely small.

The evolution of nonhydrostatic stresses (differential stress $t$ ) in $\mathrm{Nb}$ compressed in a diamond anvil cell (DAC) with different pressure-transmitting media has been studied. ${ }^{10}$ With a low shear modulus (38 GPa) at ambient pressure and a small pressure coefficient of the shear modulus (0.44), $\mathrm{Nb}$ is not expected to have large $t$. However, because of high elastic anisotropy, ${ }^{11}$ the diffraction data exhibit large nonhydrostatic-pressure effects even though the $t$-values are small. ${ }^{5,10}$ The diffraction data from $\mathrm{Nb}$ samples compressed with helium pressure medium show the presence of small nonhydrostatic stresses. ${ }^{5}$ The earlier measurements of $t$ used the diffraction data recorded with the conventional diffraction geometry wherein the incident $\mathrm{x}$-ray beam passes parallel to the load axis of the DAC ${ }^{5,10}$ The determination of $t$ from the diffraction data recorded with this geometry

\footnotetext{
a) Authors to whom correspondence should be addressed. Electronic addresses: aksingh@nal.res.in and singhnal@yahoo.com.
}

requires the knowledge of a parameter $\alpha$, which is generally assumed to lie between 0.5 and 1 . If $\alpha$ is confined within this range, then the $t$ derived from the diffraction data with $\alpha=1$ gives the lower bound. ${ }^{10,12-15}$ However, a recent analysis showed that the correct value of $\alpha$ is in the range $0<\alpha<1$ or $1<\alpha<2$ depending on whether the anisotropy factor $x$ is greater or less than $1 .{ }^{16}$ Here, $x=2 C_{44} /\left(C_{11}-C_{12}\right)$, and $C_{i j}$ denote single-crystal elastic moduli. Since, $x<1$ for $\mathrm{Nb}$, we expect $\alpha>1$, and the strength $t$ determined with $\alpha=1$ in earlier studies ${ }^{5,10}$ can no longer be taken to represent the lower bound. In this study, we determined $t$ as function of pressure without any ambiguity arising from the uncertainty in the correct value of $\alpha$. We used boron-epoxy gaskets to contain $\mathrm{Nb}$ sample and a DAC to pressurize the sample. The diffraction patterns were recorded at each pressure using the radial diffraction geometry wherein the monochromatic $\mathrm{x}$-ray beam incident on the sample is perpendicular to the load direction of the DAC. The $t$-values derived from the diffraction data in this geometry do not require the knowledge of $\alpha .^{13-15}$ However, the $C_{i j}$-values derived from the diffraction data obtained with radial geometry are sensitive to the choice of $\alpha^{13-16} \mathrm{We}$ derived $C_{i j}$ at each pressure for different values of $\alpha$ and noticed that for a threshold value of $\alpha=\alpha_{0}$, the $C_{i j}$ are in excellent agreement with those obtained from extrapolation using the one-atmosphere elastic moduli $C_{i j}(0)$ and the pressure derivatives $C_{i j}^{\prime}(0)$. The parameter $\alpha_{0}$ is found to decrease with increasing pressure.

\section{EXPERIMENTAL DETAILS}

The Nb sample from Sigma-Aldrich, Milwaukee, USA was in form a rod of $12.7 \mathrm{~mm}$ diameter. The sample purity was better than $99.9 \%$ with Ta (700 ppm) and Si (180 ppm) as the major impurities. The diamond anvil cell (DAC) used 
for these experiments had diamond anvils with $300 \mu \mathrm{m}$ culets. The experiments were performed on the beamline 16 BMD at the High-Pressure Collaboration Access Team (HPCAT) at the Advanced Photon Source (APS). The crosssectional area and wavelength of the incident beam were $15 \times 15 \mu \mathrm{m}^{2}$ and $0.040847 \mathrm{~nm}$, respectively. Amorphous boron-epoxy gaskets of $400 \mu \mathrm{m}$ diameter and $50 \mu \mathrm{m}$ thickness with a $50 \mu \mathrm{m}$ central hole were used to contain the sample. The details of the gasket fabrication can be found in an article by Merkel and Yagi. ${ }^{17}$ A small piece $\left(40 \times 40 \mu \mathrm{m}^{2}\right)$ was cut from the as-supplied $\mathrm{Nb}$ sample and placed in the central hole. To ensure maximum nonhydrostatic stresses, the sample was compressed without any pressure-transmitting medium. The radial geometry (incident $\mathrm{x}$-ray beam perpendicular to the load axis of the DAC) was used. The diffraction patterns were recorded on a Mar345 online flat image-plate. Conical openings $\left(70^{\circ}\right)$ perpendicular to the compression axis of the DAC allowed incident and diffracted beams to pass through the DAC.

The $d$-spacings as a function of azimuth angle were determined from the radial diffraction images using the software package Multifit 4.2. It provides decomposition of $2 \mathrm{D}$ diffraction images into azimuthal slices using fit $2 \mathrm{~d}$ and fits peak positions as well as intensities of the diffraction peaks. We used this software to integrate the diffraction images over $5^{\circ}$-segments of the azimuth angle, resulting in 72 slices. The fitted peak positions were used to calculate the $d$-spacings as a function of azimuth angle.

\section{THEORY}

In the development of the lattice strain theory, the stress state in the sample compressed in a DAC without any pressure transmitting medium is described by an axially symmetric stress tensor with the diagonal terms $\sigma_{11}, \sigma_{11}$, and $\sigma_{33}$, and the off-diagonal terms are taken to be zero. The component $\sigma_{33}$ is along the load direction of the DAC. The $d$-spacing measured on the sample compressed in a DAC is given by $^{12-16}$

$$
d_{m}(h k l)=d_{P}(h k l)\left[1+\left(1-3 \cos ^{2} \psi\right) Q(h k l)\right] .
$$

$d_{P}(h k l)$ is the $d$-spacing under the mean normal stress (equivalent hydrostatic pressure) $\sigma_{P}=\left(2 \sigma_{11}+\sigma_{33}\right) / 3$. The $d_{m}(h k l)$ versus $\left(1-3 \cos ^{2} \psi\right)$ plot is a straight line, and the slope and intercept of the line give $\left[d_{P}(h k l)\right] Q(h k l)$ and $d_{P}(h k l)$, respectively. ${ }^{14}$ For the cubic system, $d_{m}(h k l)$ and $d_{P}(h k l)$ can be replaced by $a_{m}(h k l)$ and $a_{P}$, respectively. It may be noted that $a_{P}$ is the unit cell parameter under hydrostatic pressure $\sigma_{P}$, and therefore, is independent of $h k l$. The parameter $\psi$ denotes the angle between the diffraction vector and load axis of the DAC. $Q(h k l)$ is given by

$$
Q(h k l)=t / 6 G^{X}(h k l) .
$$

Here, $t=\left(\sigma_{33}-\sigma_{11}\right)$. The use of von Mises yield criterion suggests that $t \leq \sigma_{Y}$, where $\sigma_{Y}$ is the yield strength of the sample material under pressure $\sigma_{P} . G^{X}(h k l)$ denotes the diffraction shear modulus for the set of planes $(h k l)$. It is assumed $^{13,14}$ that $G^{X}(h k l)$ is adequately described by a relation of the form

$$
\left[G^{X}(h k l)\right]^{-1}=\left\{\alpha\left[G_{R}^{X}(h k l)\right]^{-1}+(1-\alpha)[G(V)]^{-1}\right\} .
$$

The parameter $\alpha$ determines relative weights of the two kinds of shear moduli. $G_{R}^{X}(h \mathrm{kl})$ denotes the diffraction shear modulus calculated under the assumption of stress continuity across the grain boundaries, the averaging being done only over the crystallites that contribute to the diffracted intensity at the point of observation. For the cubic system,

$$
\left[G_{R}^{X}(h k l)\right]^{-1}=2\left[\left(S_{11}-S_{12}\right)-3\left(S_{11}-S_{12}-S_{44} / 2\right) \Gamma(h k l)\right] .
$$

The $S_{i j}$ denote the single-crystal elastic compliances and

$$
\Gamma(h k l)=\left(h^{2} k^{2}+k^{2} l^{2}+l^{2} h^{2}\right) /\left(h^{2}+k^{2}+l^{2}\right)^{2} .
$$

$G(V)$ is the aggregate shear modulus of the polycrystalline solid containing randomly oriented crystallites calculated under the assumption that the strain is continuous across the interfaces between the crystallites. ${ }^{18}$

The terms $d_{P}(h k l)$ and $Q(h k l)$ can be determined from Eq. (1) using $d_{m}(h k l)$ versus $\psi$ data. The parameter $t$ can be computed using the relation ${ }^{14}$

$$
t=6 G<Q(h k l)>f,
$$

where

$$
\begin{gathered}
f=A / B \\
A=\{[(2 x+3) / 10]+5 x /[2(3 x+2)]\} \\
B=\{\alpha[x-(x-1)<3 \Gamma(h k l)>]+5 x(1-\alpha) /(3 x+2)\} \\
x=2\left(S_{11}-S_{12}\right) / S_{44}=2 C_{44} /\left(C_{11}-C_{12}\right) .
\end{gathered}
$$

The symbol $<>$ denotes the average over all crystallographic reflections, and $G$ is the aggregate shear modulus. It is found that $f \cong 1$ for the $x$-values exhibited by real solids and a wide range of $\alpha$-values. Experimental $G^{X}(h k l)$ denoted by $\left[G^{X}(h k l)\right]_{m}$ can be obtained from the measured $Q(h k l)$ and $t$ from Eq. (2). Eqs. (2)-(5) suggest that $Q(h k l)$ versus $3 \Gamma(h k l)$ plot is a straight line with intercept $m_{0}$ and slope $m_{1}$ given by ${ }^{14}$

$$
\begin{gathered}
m_{0}=(\alpha t / 3)\left\{S_{11}-S_{12}+\frac{5}{2}\left(\alpha^{-1}-1\right) \frac{\left(S_{11}-S_{12}\right) S_{44}}{3\left(S_{11}-S_{12}\right)+S_{44}}\right\} \\
m_{1}=-(\alpha t / 3)\left(S_{11}-S_{12}-S_{44} / 2\right) .
\end{gathered}
$$

The single-crystal elastic compliances (and elastic moduli by inversion of the compliance matrix) can be determined ${ }^{13-15}$ if Eqs. (7a) and (7b) are supplemented with the information on the pressure-dependent compressibility given by

$$
S_{11}+2 S_{12}=1 / 3 K
$$

Kröner ${ }^{19}$ derived a rigorous equation to compute from the single-crystal elastic moduli the shear modulus of the 
isotropic aggregate of crystallites of the cubic system. The Kröner-equation of the following form gives aggregate shear modulus $G$ in terms of single-crystal elastic moduli $C_{i j}$

$$
G^{3}+a G^{2}+b G+c=0 .
$$

The parameters in Eq. (9) are given by

$$
\begin{gathered}
a=(3 / 40)\left[15 K+(20 / 3) C^{\prime}\right], \\
b=-(3 / 8)\left(K C_{44}+4 C^{\prime} C_{44}\right), \\
c=-(3 / 4) K C^{\prime} C_{44} .
\end{gathered}
$$

Here, $K=\left(C_{11}+2 C_{12}\right) / 3$ and $C^{\prime}=\left(C_{11}-C_{12}\right) / 2$. de Wit ${ }^{17}$ showed that $G=G^{X}(h k l)$ if the following values of $a$, $b$, and $c$ are used in Eq. (9),

$$
\begin{gathered}
a=(3 / 40)\left\{15 K+[12-60 \Gamma(h k l)] C_{44}\right. \\
\left.-[(16 / 3)-60 \Gamma(h k l)] C^{\prime}\right\}, \\
b=(3 / 40)\left\{10[1-3 \Gamma(h k l)-(9 / 10)] K C_{44}\right. \\
\left.+[30 \Gamma(h k l)-6] K C^{\prime}-20 C^{\prime} C_{44}\right\},
\end{gathered}
$$

and

$$
c=-\frac{3}{4} K C_{44} C^{\prime} .
$$

The terms in the original equations of Kröner ${ }^{19}$ and of de $\mathrm{Wit}^{20}$ are rearranged to obtain Eqs. (9), (10a)-(10c), (11a)(11c). The $G^{X}(h k l)$ at high pressure can be computed by using pressure-dependent $C_{i j}$. The computed diffraction shear moduli, $G^{X}(h k l)_{e l .}$, are under small deformation (elastic strain). The measured diffraction shear moduli, $G^{X}(h k l)_{m}$, determined from Eq. (2) contains the effect of plastic deformation of the sample. A comparison of the two gives an idea of the magnitude of this effect.

The high-pressure elastic moduli can be computed from the ambient pressure elastic moduli and their pressure derivatives using Birch equation ${ }^{21}$ of the form

$$
C_{i j}=C_{i j}(0) y^{5 / 3}\left\{1+\frac{1}{2}\left[3 K(0) C_{i j}^{\prime}(0) / C_{i j}(0)-5\right]\left(y^{2 / 3}-1\right)\right\} .
$$

The symbol (0) denotes the quantity at zero (ambient) pressure. The prime indicates the first pressure derivative and $y=V(0) / V$, where $V$ is the unit cell volume under pressure. The $C_{i j}(0)$ and $C_{i j}^{\prime}(0)$ for $\mathrm{Nb}$ used in this study are shown in Table I. The high-pressure shear modulus required for the computation of $t$ from Eq. (6a) taken as the arithmetic mean ${ }^{22}$ of the computed Reuss ${ }^{23}$ and Voigt ${ }^{18}$ shear moduli. The ultrasonic technique gives the adiabatic $C_{i j}$, whereas the isothermal elastic moduli are relevant to the static pressure

TABLE I. The single-crystal elastic moduli (GPa) and pressure derivatives of $\mathrm{Nb}$ (Ref. 11) used in the analysis of the diffraction data.

\begin{tabular}{lclc}
\hline \hline$C_{11}(0)$ & 246.2 & $C_{44}(0)$ & 28.7 \\
$C_{11}^{\prime}(0)$ & 5.22 & $C_{44}^{\prime}(0)$ & 0.281 \\
$C_{12}(0)$ & 132.9 & $K_{T}(0)$ & 169 \\
$C_{12}^{\prime}(0)$ & 3.37 & $K_{T}^{\prime}(0)$ & 4.02 \\
\hline \hline
\end{tabular}

measurements. The isothermal bulk modulus $K$ is derived by dividing the adiabatic $K$ by a term $\left(1+\alpha_{V} \gamma T\right)$, where $\alpha_{V}, \gamma$, and $T$ are the coefficient of volume expansion, Gruneissen parameter, and temperature in Kelvin, respectively. The quantities $G$ and $S$ are invariant under adiabatic to isothermal conversion. ${ }^{24}$ It may be noted that, in the present pressure range, the high-pressure $C_{i j}$ computed from Eq. (12) do not differ much from the corresponding values obtained from linear extrapolation. For example, at $50 \mathrm{GPa}, C_{11}$ and $C_{12}$ obtained from linear extrapolation are $\sim 5 \%$ higher than those obtained from Eq. (14), whereas the difference is $\sim 7 \%$ for $C_{44}$.

\section{RESULTS AND DISCUSSION}

\section{A. General}

The diffraction patterns of $\mathrm{Nb}$ at $33.2 \mathrm{GPa}$ are shown in Fig. 1. The diffraction angle $2 \theta$ as function of azimuth $\delta$ is shown in Fig. 2. In absence of any nonhydrostatic stress, i.e., $t=0$ ), the diffraction rings recorded on a flat area-detector placed perpendicular to the incident beam are circles and the $2 \theta$ versus $\delta$ plots are straight lines. For nonzero $t$, the diffraction rings are distorted. This distortion is seen clearly in Fig. 2. The angle $\psi$ that appears in Eq. (1) can be computed using the following relation:

$$
\cos \psi=\cos \theta \cos \delta .
$$

The point at which the plane containing the load axis of the DAC and the incident $\mathrm{x}$-ray beam intersects the diffraction ring is taken as $\delta=0$.

The form of Eq. (1) suggests that $d_{m}(h k l)$ versus $\psi$ data derived from any one quadrant of the diffraction patterns

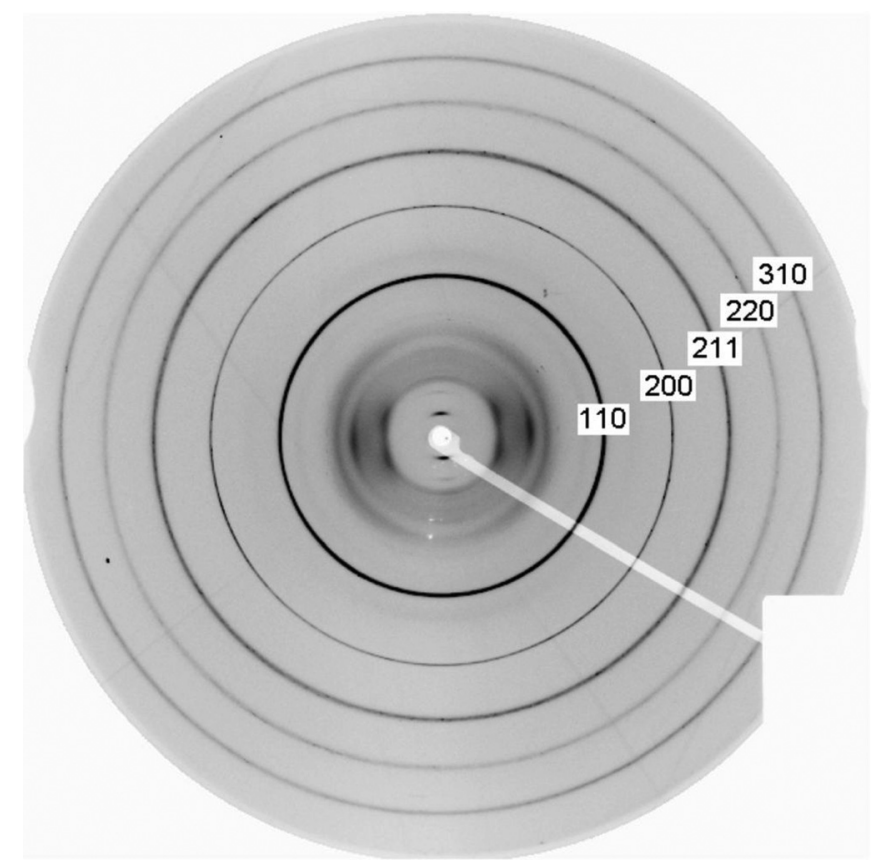

FIG. 1. The diffraction pattern from $\mathrm{Nb}$ at $33.2 \mathrm{GPa}$. The diffuse rings inside the 110 reflection are from amorphous boron and epoxy used in the fabrication of the gasket. Circular shadow at the center of the pattern is cast by the primary-beam stop and line shadow is produced by the support wire of the beam stop. 


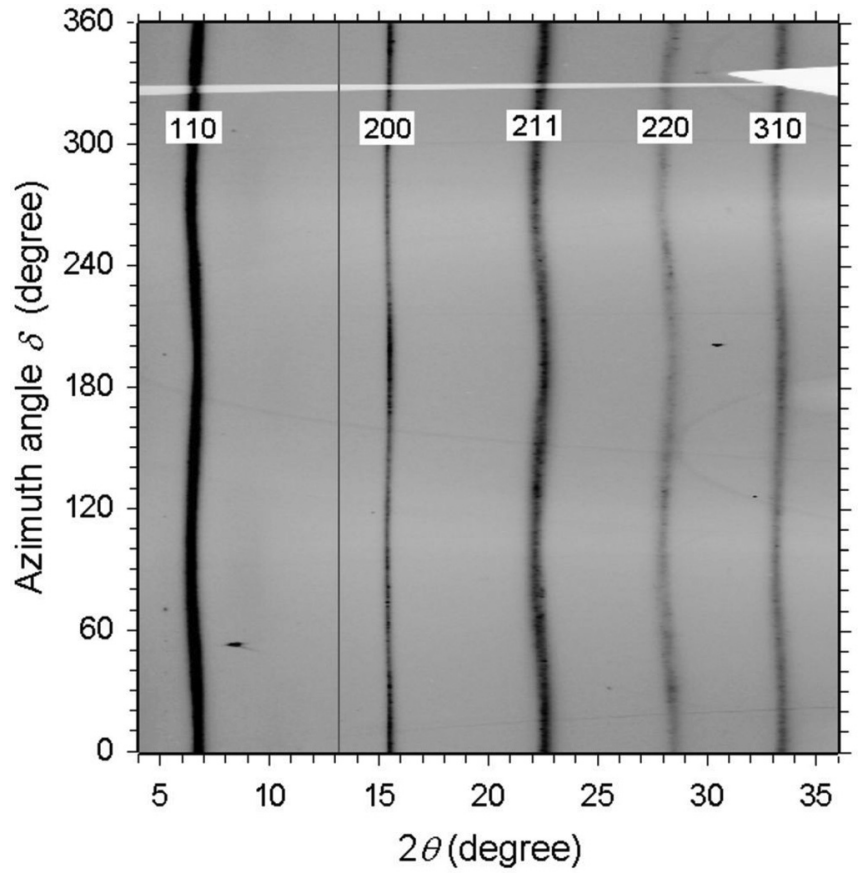

FIG. 2. Diffraction rings of Fig. 1 converted to Cartesian coordinates $\delta$ vs $2 \theta$. The traces, which should be straight lines for sample under hydrostatic pressure, are distorted in presence of nonhydrostatic stresses.

contain all the information about the strength and elasticity. However, additional information about the stress state can be obtained if $d_{m}(h k l)$ versus $\psi$ data derived from full diffraction rings are available. Figure 3 shows $d_{m}(h k l)$ versus $\left(1-3 \cos ^{2} \psi\right)$ plots for the first five reflections. The data are shown by different symbols for $\delta$ in different quadrants. In an ideal case, the data in four quadrants should lie on a single straight line. However, the data show systematic deviations from straight line. The filled and unfilled symbols fall on curves with opposite curvatures forming a loop. Such trends in the $d_{m}(h k l)$ versus $\left(1-3 \cos ^{2} \psi\right)$ plots arise due to the presence of the off-diagonal components in the stress tensor. $^{25,26}$ All points were given equal weights and a straight line was fitted. The $Q(h k l)$-values for the five observed reflections were determined from the slopes and intercepts of these lines. The parameter $d_{P}(h k l)$ is introduced in the lattice strain theory as the d-spacing under equivalent hydrostatic pressure (mean normal stress) and therefore $a_{P}(h k l)$ derived from $d_{P}(h k l)$ for the cubic system should be independent of the $h k l$. Figure 4 shows the standard errors calculated from $a_{P}(h k l)$ of five reflections for all pressure runs. As expected from the theory, $a_{P}$ calculated from different reflections are independent of $h \mathrm{kl}$ within the errors of measurements. The pressures were calculated from the measured volume compression computed from $a_{P}$ using Birch equation. The one atmosphere bulk modulus and its pressure derivative used for the calculation of pressures are given in Table I. Figure 5 shows $Q(h \mathrm{kl})$ of different pressure runs. The values of $Q(110)$ and $Q(220)$ should be identical. The observed differences are small and are within the errors of measurement. Figure 6 shows the $Q(h k l)$ versus $3 \Gamma(h k l)$ plots for two pressures and the straight lines through the experimental points. Figure 7 show the intercept $m_{0}$ on the and slope $m_{1}$ for all pressure runs.

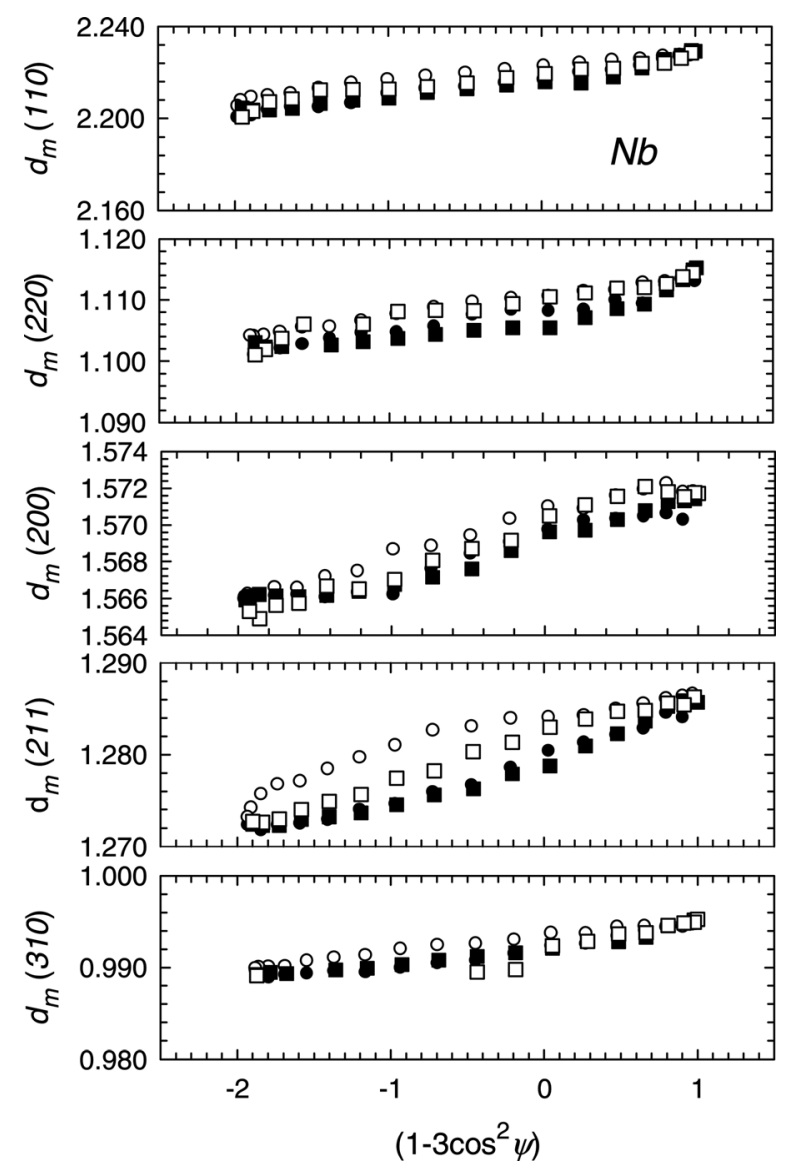

FIG. 3. The $d$-spacings as a function of $\left(1-3 \cos ^{2} \psi\right)$ corresponding to Fig. 1. Different ranges of $\psi$ are shown by different symbols. Filled circles, $\theta \rightarrow 90^{\circ}$; unfilled circles, $90^{\circ} \rightarrow\left(180^{\circ}-\theta\right)$; filled square, $\left(180^{\circ}+\theta\right) \rightarrow 270^{\circ}$, unfilled square, $270^{\circ} \rightarrow(360-\theta)$.

\section{B. Estimation of $t$}

The compressive strength $t$ is computed from Eq. (6a) assuming $f=1$. The validity of this assumption in the present case is discussed in Sec. IV E. Here, $\langle Q(h k l)\rangle$ represents the arithmetic mean of $Q(h k l)$ of five observed reflections. The aggregate shear modulus at high pressure was computed from extrapolated single-crystal elastic moduli. Three

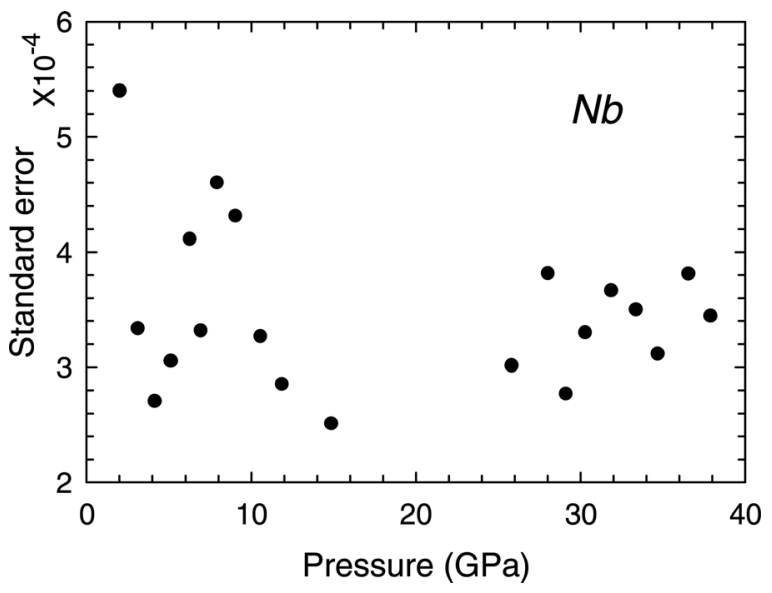

FIG. 4. Standard errors computed from $a_{P}$ of 5 reflections for all pressure runs. Small magnitudes of the standard errors indicate that $a_{P}$, as expected from the theory, is independent of $h k l$. 


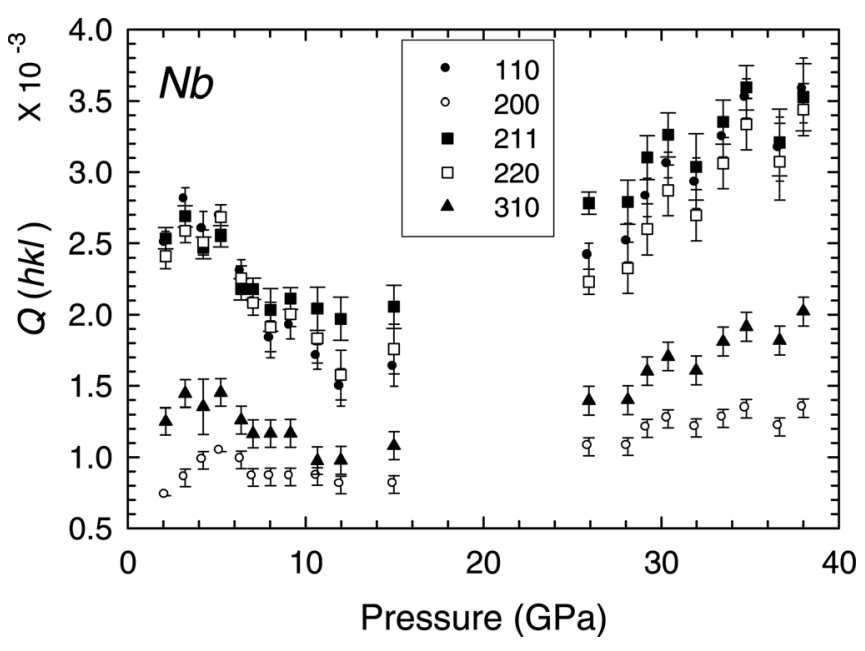

FIG. 5. $Q(h k l)$ of first five reflections for all the runs.

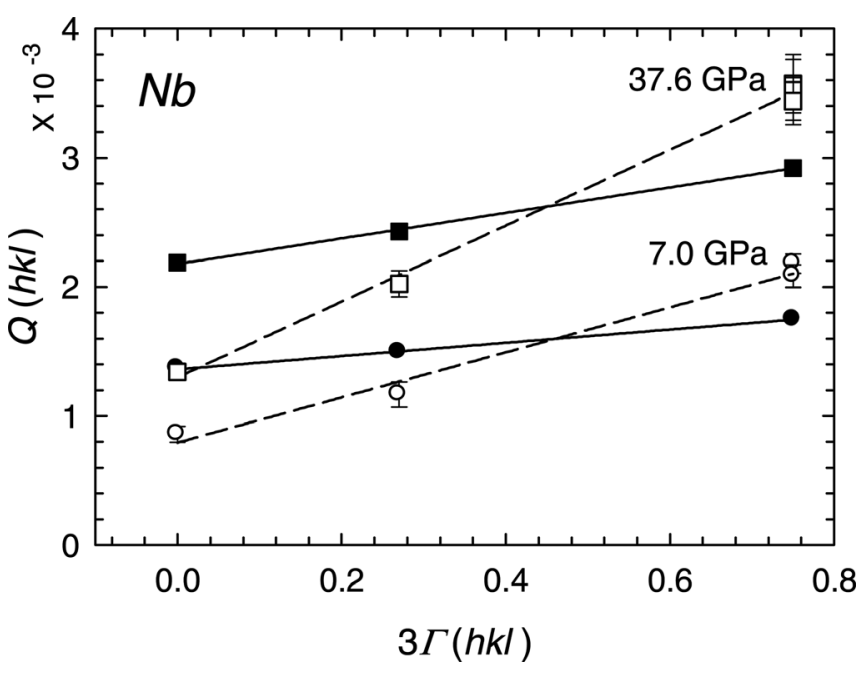

FIG. 6. $Q(h k l)$ vs $3 \Gamma(h k l)$ plots for the runs at 7 and $37.6 \mathrm{GPa}$. Unfilled symbols - experimental data. Filled symbols - computed from Eq. (2) using measured $t$ and $G^{X}(h k l)_{e l \text {. }}$

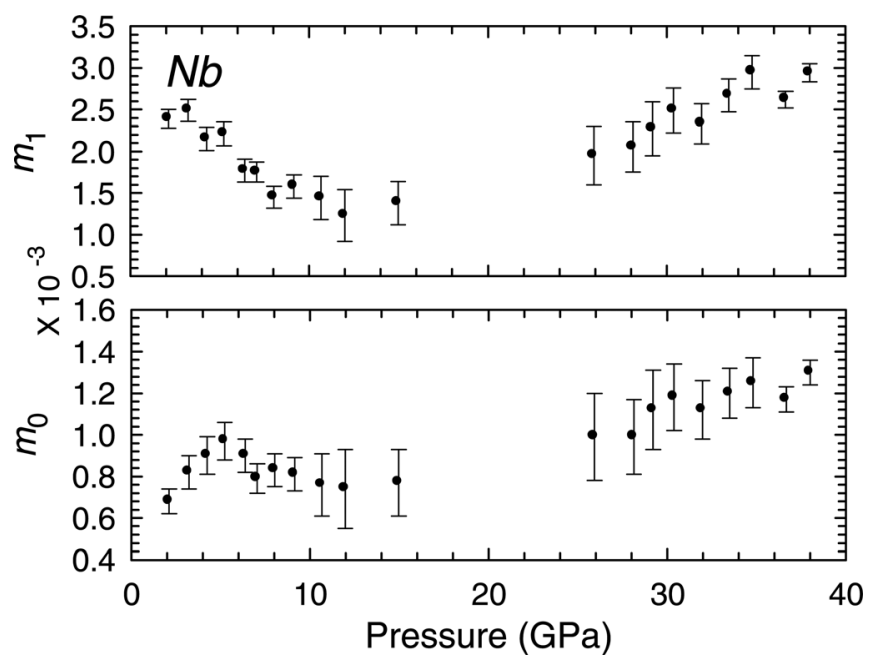

FIG. 7. Plots showing the intercept $m_{0}$ and slope $m_{1}$ of the straight lines drawn through the $Q(h k l)$ vs $3 \Gamma(h k l)$ data. procedures, namely, Voigt-Reuss-Hill (V-R-H) average, ${ }^{18,22,23}$ Hashin-Shtrikman (H-S) method, ${ }^{27,28}$ and Kröner method ${ }^{19}$ [Eqs. (9) and (10)], are available in the literature for computing aggregate shear moduli from the single-crystal elastic moduli. The aggregate shear moduli of $\mathrm{Nb}$ computed at 7 and $37.6 \mathrm{GPa}$ (the highest pressure in this study) by these procedures are listed in Table II. The shear moduli computed by V-R-H and H-S methods show maximum difference but is only $\sim 0.2 \%$. We used extrapolated $C_{i j}$ to compute aggregate shear moduli at different pressures by $\mathrm{V}-\mathrm{R}-\mathrm{H}$ method. Figure 8 (a) shows the $t-P$ data. The measurements (standard tensile tests) at ambient pressure and temperature show that the yield stress is sensitive to the impurity levels. ${ }^{29}$ The samples with an impurity level 1500 ppm atom fraction is $0.08 \mathrm{GPa}$ and increases to $0.21 \mathrm{GPa}$ for samples with impurity level $6500 \mathrm{ppm}$ atom fraction. ${ }^{29}$ There are no strength data below $2 \mathrm{GPa}$ in the present measurements. We take tensile test data at ambient pressure to populate the low-pressure range. These data points are also shown in Fig. 8(a). Combining data from the tensile test with the present data that are under compressive loads is justified as the stress-strain curves of metals and alloys under tension and compression are nearly indistinguishable. ${ }^{30}$ It is seen that initially the strength increases steeply with increasing pressure, reaching a maximum at $\sim 5 \mathrm{GPa}$. With further increase in pressure, the strength decreases until the pressure reaches $\sim 12 \mathrm{GPa}$ and exhibits a monotonic increase at higher pressures.

In an earlier study, ${ }^{10}$ herein referred to as ST (Singh and Takemura), $t$-values were reported in $\mathrm{Nb}$ samples compressed in a DAC [Fig. 8(b)]. Large $t$-values were observed despite the use of pressure-transmitting medium (PTM) because the sample bridged the anvils in the runs with helium PTM. In case of the runs with methanol-ethanol-water mixture, the combined effects of bridging and the strength of PTM contributed to large $t$. The shape of the $t-P$ curve is similar to that in the present study. However, large quantitative differences exist between the two sets of data. The maximum and minimum in the $t$ - $P$ plot [Fig. 8(b)] occur at $\sim 30$ and $\sim 75 \mathrm{GPa}$, respectively. These pressures differ from those observed in the present study by a factor $\sim 6$. Further, the $t$-values observed by ST are significantly larger than those observed in the present study. For example, $t$ at $33 \mathrm{GPa}$ is larger than the present value by a factor $\sim 3$. It must be noted that ST derived the $t$-values from the diffraction data taken with conventional geometry wherein the incident $\mathrm{x}$-ray beam is parallel to the load axis. The $d_{m}(h k l)$ versus

TABLE II. Comparison of aggregate shear modulus (GPa) of Nb computed from single-crystal elastic moduli at high pressure by different methods.

\begin{tabular}{lcc}
\hline \hline$P$ & $7.0 \mathrm{GPa}$ & $37.6 \mathrm{GPa}$ \\
\hline $\mathrm{G}(\mathrm{V}-\mathrm{R})^{\mathrm{a}}$ & 41.03 & 53.17 \\
$\mathrm{G}(\mathrm{H}-\mathrm{S})^{\mathrm{b}}$ & 41.133 & 53.328 \\
$\mathrm{G}(\mathrm{K})^{\mathrm{c}}$ & 41.070 & 53.198 \\
\hline \hline
\end{tabular}

${ }^{a}$ Arithmetic mean of Voigt and Reuss bounds (Refs. 18, 31, 32).

${ }^{\mathrm{b}}$ Mean of Hashin and Shtrikman bounds (Refs. 37, 38).

${ }^{\mathrm{c}}$ Kröner method Eqs. (9) and (10). 


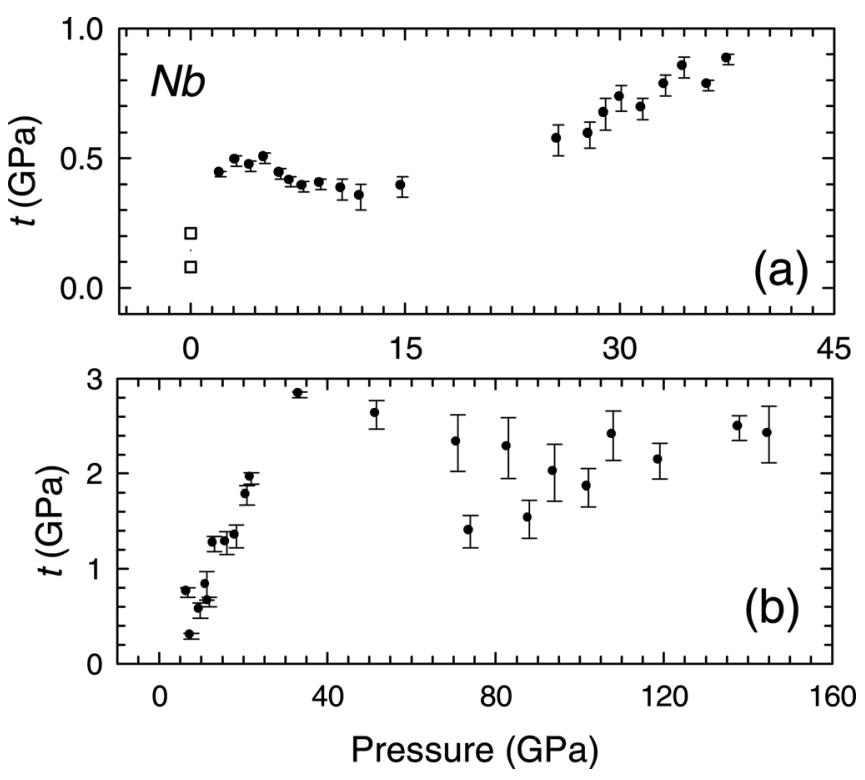

FIG. 8. (a) The parameter $t$ as function of pressure. Filled circles - parameter $t$; Unfilled squares - yield stress at ambient pressure (Ref. 29). (b) $t$ vs pressure data from Ref. 10.

$3\left(1-3 \sin ^{2} \theta\right) \Gamma(h k l)$ plot for the diffraction data recorded with this geometry is a straight line and $t$ is given by

$$
t \cong-3 M_{1} /\left(\alpha S M_{0}\right)
$$

Here, $M_{0}$ and $M_{1}$ are the intercept and slope of the line, respectively. The $t$-values reported by ST are calculated from Eq. (14) with $\alpha=1$. It is now known that $\alpha>1$ for $\mathrm{Nb}$ in presence of plastic deformation of the sample (see Sec. IV D). Therefore, the $t$-values reported by ST are gross overestimates. We recalculated the $t$-values from the data of ST using the present $\alpha$-values in Eq. (14). These $t$-values are in better agreement with the present data but still large differences persist. For example, recalculated $t$ at $33 \mathrm{GPa}$ is $\sim 2 \mathrm{GPa}$ as compared to the present value of $\sim 1 \mathrm{GPa}$. However, the use of $\alpha$-value obtained from the present study in the analysis of data of ST is not justified because the extents of plastic deformation at a given pressure are different in two experiments and would lead to different $\alpha$-values. As seen from the experiments at ambient pressure, ${ }^{29}$ the yield strength is sensitive to the impurities in the $\mathrm{Nb}$ sample. Though $\mathrm{Nb}$ samples in two studies have $99.9 \%$ nominal purity, a slightly higher impurity level in the sample used by ST can result in significantly higher yield stress. A meaningful comparison of the $t$ - $P$ data in the two studies is difficult because of these factors.

\section{Diffraction shear modulus}

The significance of diffraction shear modulus has been discussed in an earlier article. ${ }^{16}$ The diffraction shear modulus $G^{X}(h k l)_{e l}$. computed using Eqs. (9) and (11) is under small (elastic) deformation. Further, Eqs. (9)-(11) have been derived assuming random orientation of the crystallites in the sample. The experimental diffraction shear modulus $G^{X}(h k l)_{m}$ is obtained from the high-pressure diffraction data using Eq. (2) with experimental $Q(h k l)$ and $t$. The compres- sion of the sample in a DAC is accompanied by considerable plastic deformation and attendant texture in the sample. As discussed in Sec. IV A, the diffraction data indicate the presence of small off-diagonal terms in the stress tensor, a factor not considered in the development of the lattice strain theory. For these reasons, $G^{X}(h k l)_{m}$ is expected to differ from $G^{X}(h k l)_{e l}$. Table III gives a comparison of $G^{X}(h k l)_{m}$ and $G^{X}(h k l)_{e l}$ at 7 and $37.6 \mathrm{GPa}$. In an earlier study on this topic, the $Q(h k l)_{m}$ derived from the diffraction data were compared with $Q(h k l)_{e l}$. derived by using $G^{X}(h k l)_{e l}$. and $t$ obtained from the diffraction data in Eq. (2). ${ }^{16}$ The $Q(h k l)_{e l}$. versus $3 \Gamma(h k l)$ plots are shown in Fig. 6 together with $Q(h k l)_{m}$ versus $3 \Gamma(h k l)$ data. It is seen that data points for both $Q(h k l)_{m}$ and $Q(h k l)_{e l}$. lie on straight lines with positive slopes. This is expected as $S$ is negative $(x<1)$ for $\mathrm{Nb}$. The magnitude of the slope of the line through the $Q(h k l)_{m}$-points is larger than the slope of the line through the $Q(h k l)_{e l}$-points. Similar trends were observed in case of $\mathrm{FeO}$ and $\mathrm{Mo}$, which have negative $S .{ }^{16}$ It is to be noted that the trends are reversed in case of $\mathrm{Au}$ and bcc-Fe, both with positive $S .{ }^{16}$

Also given in Table III are the values for $G^{X}(h k l)$ computed from Eqs. (3) and (4) with $\alpha=0.5 . G^{X}(h k l)$ is found to agree reasonably well with $G^{X}(h k l)_{e l}$. The form of Eq. (3) may be erroneously taken to imply that $G_{R}^{X}(h k l)$ and $G(V)$ give the lower and upper bounds, respectively, of $G^{X}(h k l)$. To illustrate this point in case of $\mathrm{Nb}$ we plot in Fig. 9 different shear moduli at $7 \mathrm{GPa}$ as a function of $3 \Gamma(h k l)$. The shear modulus $G(V)$ is independent of $3 \Gamma(h k l)$. It is seen that for $3 \Gamma(h k l)$ less than a critical value $(\sim 0.42$ at $7 \mathrm{GPa}$ for $\mathrm{Nb}), G_{R}^{X}(h k l)>G(V)$. Above the critical value of $3 \Gamma(h k l)$, $G(V)>G_{R}^{X}(h k l)$. However, in the entire range of $3 \Gamma(h k l)$, the average of $G_{R}^{X}(h k l)$ and $G(V)$ gives $G^{X}(h k l)$, which is close to $G^{X}(h k l)_{e l}$. Thus, $G_{R}^{X}(h k l)$ and $G(V)$ do not bound $G^{X}(h k l)$ in the sense $G(R)$ and $G(V)$ bound the aggregate shear modulus, even though the average of the two $(\alpha=0.5)$ gives a value close to $G^{X}(h k l)_{e l}$. This, however, should not be considered a violation of Hill theorem that applies to aggregate shear modulus. ${ }^{22}$ The computation of aggregate shear moduli $G(R)$ involves averaging overall possible orientations whereas $G_{R}^{X}(h k l)$ is computed by taking average over

TABLE III. A comparison of computed and measured diffraction shear moduli (GPa) for $\mathrm{Nb}$ at $V(0) / V$-values of 1.0391 and 1.1745 , the respective pressures being 7 and $37.6 \mathrm{GPa}$.

\begin{tabular}{lllllll}
\hline \hline $\mathrm{DSM}^{\mathrm{a}}$ & \multicolumn{1}{c}{$h k l$} & 110 & 200 & 211 & 220 & 310 \\
\hline$G^{X}(h k l)_{e l .}{ }^{\mathrm{b}}$ & & 39.106 & 50.036 & 39.106 & 39.106 & 45.776 \\
$G^{X}(h k l)_{m}{ }^{\mathrm{c}}$ & $7 \mathrm{GPa}$ & 32.8 & 79.5 & 31.4 & 32.8 & 58.7 \\
$G^{X}(h k l)^{\mathrm{d}}$ & & 39.33 & 53.27 & 39.33 & 39.33 & 46.27 \\
$G^{X}(h k l)_{e l .}{ }^{\mathrm{b}}$ & & 50.222 & 67.053 & 50.222 & 50.222 & 60.422 \\
$G^{X}(h k l)_{m}{ }^{\mathrm{c}}$ & $37.6 \mathrm{GPa}$ & 40.1 & 109.2 & 41.6 & 42.7 & 72.5 \\
$G^{X}(h k l)^{\mathrm{d}}$ & & 50.73 & 72.83 & 50.73 & 50.73 & 61.10 \\
\hline \hline
\end{tabular}

${ }^{\mathrm{a}}$ Diffraction shear modulus.

${ }^{\mathrm{b}}$ Computed from Eqs. (9) and (11). Elastic moduli required in computation were obtained by extrapolation to the relevant compression using Eq. (14).

${ }^{\mathrm{c}}$ Obtained from Eq. (2) with experimental values of $t$ and $Q(h k l)$.

${ }^{\mathrm{d}}$ Computed from Eqs. (3) and (4) using did yo $\alpha=0.5$ and extrapolated elastic moduli. 


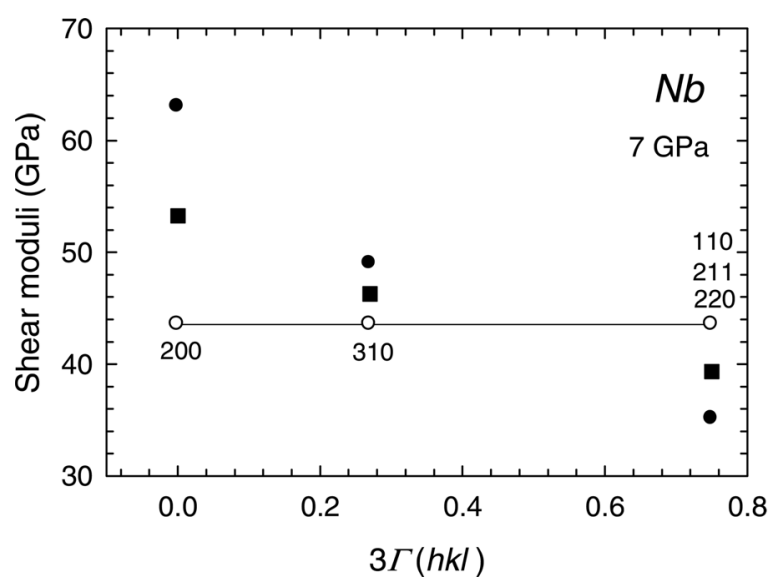

FIG. 9. Different shear moduli at $7 \mathrm{GPa}$ as functions of $3 \Gamma(h k l)$. $G(V)$ unfilled circles, $G_{R}^{X}(h k l)$ - filled circles, and diffraction shear modulus $G^{X}(h k l)$ - filled squares.

limited orientations. A more detailed discussion on this topic can be found in earlier articles. ${ }^{16,31,32}$

\section{Estimation of $\alpha_{0}$}

In this section we estimate the value of $\alpha\left(=\alpha_{0}\right)$ that gives best overall agreement with extrapolated $C_{i j}$. As discussed in Sec. III, Eqs. (7a,b) and (8) can be used to determine $C_{i j}$ if $\alpha$ is known. In the past, the diffraction data have been interpreted assuming that the parameter $\alpha$ lies between 0.5 and $1 .^{12-15,33,34}$ In a recent study, it is shown that for solids with $x<1, \alpha>1$ is required in Eqs. (7a,b) to obtain $C_{i j}$ that are in good agreement with the extrapolated $C_{i j} .{ }^{16}$ Following this trend, $\alpha>1$ is expected in case of $\mathrm{Nb}$ as $x<1$. We determined $C_{i j}$ for different $\alpha$-values and compared these with the extrapolated $C_{i j}$ using the following relation

$$
\Delta C_{i j}=\left[\left(C_{i j}\right)_{m}-C_{i j}\right] \times 100 / C_{i j} .
$$

The suffix $m$ denotes the parameter determined from the diffraction data. In a typical case, the dependences of $\left(C_{i j}\right)_{m}$ on $\alpha$ are shown in Fig. 10. It is seen that all elastic moduli do not match the corresponding extrapolated values at a single $\alpha$. We choose a value $\alpha_{0}$ such that the magnitudes of $\Delta C_{i j}$ for the three elastic moluli are nearly equal. These $\alpha_{0}$-values for

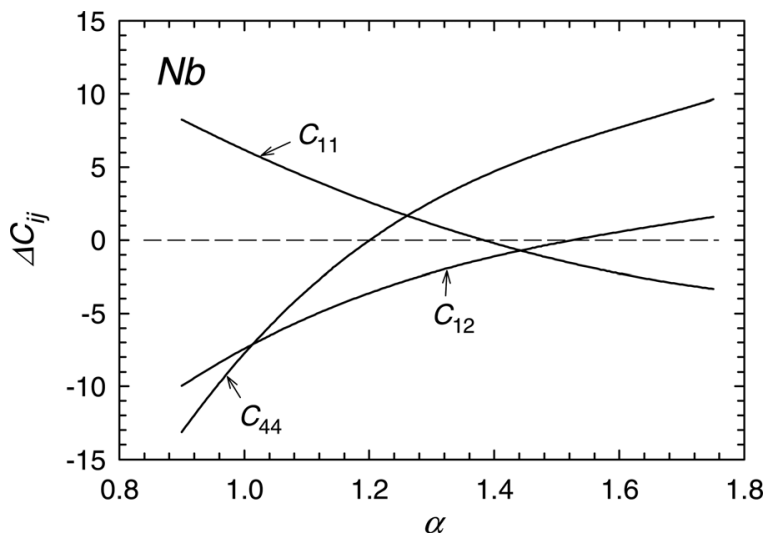

FIG. 10. The percentage deviations of $\left(C_{i j}\right)_{m}$ from extrapolated $C_{i j}$ (Eq. 15) as a function of $\alpha$. different pressure runs are found to decrease with increasing pressure (Fig. 11). The $\Delta C_{i j}$-values with $\left(C_{i j}\right)_{m}$ at $\alpha=\alpha_{0}$ are shown in Fig. 12. It is seen that $\left(C_{i j}\right)_{m}$ at $\alpha=\alpha_{0}$ match the corresponding extrapolated $C_{i j}$ within a few percent. It must be emphasized that $\alpha_{0}$ is not known a priori, and the value of $\alpha$ has to be assumed to determine $\left(C_{i j}\right)_{m}$ from the diffraction data. This leads to much larger $\Delta C_{i j}$ than those shown in Fig. 12.

It may be pointed out that $\alpha>1$ was observed earlier in experiments on $\mathrm{Fe}_{2} \mathrm{SiO}_{4}$ and $\mathrm{MgO}{ }^{31,32}$ These investigators take $\alpha>1$ to imply that the Voigt and Reuss limits do not constitute the upper and lower bounds of shear moduli when the sample undergoes plastic deformation. However, $\alpha>1$ is not observed for all the solids. Another study showed that $\alpha<1$ for $\mathrm{Au}$ and $\alpha$-iron, both with $x>1$, and $\alpha>1$ is observed only for solids with $x<1$ such as Mo and FeO. ${ }^{16}$ This study shows that for $\mathrm{Nb} \alpha>1$. This is expected based on the observation on $\mathrm{Mo}$ and $\mathrm{FeO}$ since $x<1$ for $\mathrm{Nb}$. Such a dependence of $\alpha$ on $x$ arises due to the presence of off-diagonal terms in the stress tensor, which are neglected in the derivation of lattice strain equations. ${ }^{16}$ As discussed in Sec. IV A, Fig. 3 clearly demonstrates the presence small off-diagonal terms. However, it should be kept in mind that studies on $\mathrm{Fe}_{2} \mathrm{SiO}_{4}$ and $\mathrm{MgO}^{31,32}$ are at high temperature and pressure whereas the present and an earlier study ${ }^{16}$ are at high pressure and ambient temperature. This makes a closer comparison of the two sets of studies difficult.

\section{E. Dependence of $f$ on $\alpha$}

The $t$-values have been estimated using Eq. (6a) with $f=1$. To check the validity of this assumption we computed $f$ for $\alpha$-values $1,1.5$, and 2 with different $x$-values. The results are shown in Fig. 13. The $x$-values computed from the extrapolated $C_{i j}$ suggests that it decreases from 0.50 at ambient pressure as the pressure is increased. A fit of the five-parameter double-exponential decay function to $x$ versus pressure data indicates that the limiting value of $x$ at infinite pressure is $0.267(1)$. Thus, the elastic anisotropy of $\mathrm{Nb}$ increases with increasing pressure. The dots represent $f$ computed with $\alpha_{0}$ and $x$ corresponding to the actual pressures in the experiments. It is seen that the dots lie close $f=0.975(8)$.

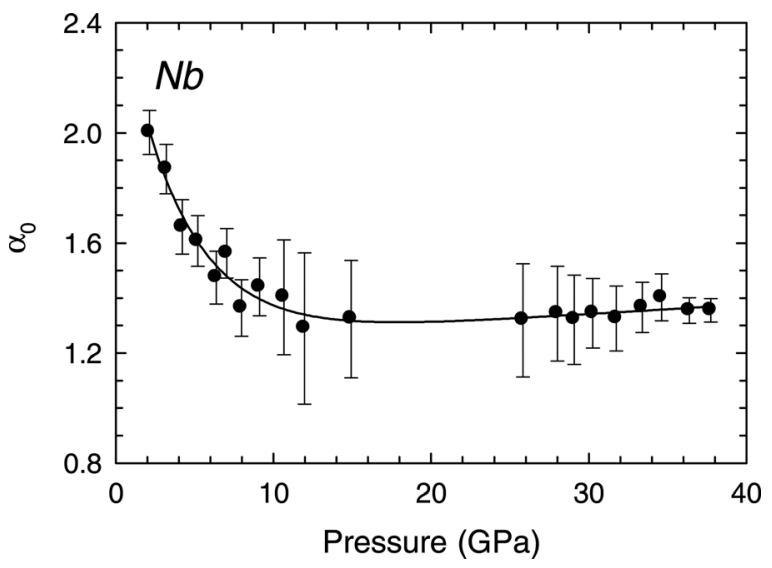

FIG. 11. Variation of $\alpha_{0}$ with pressure. 


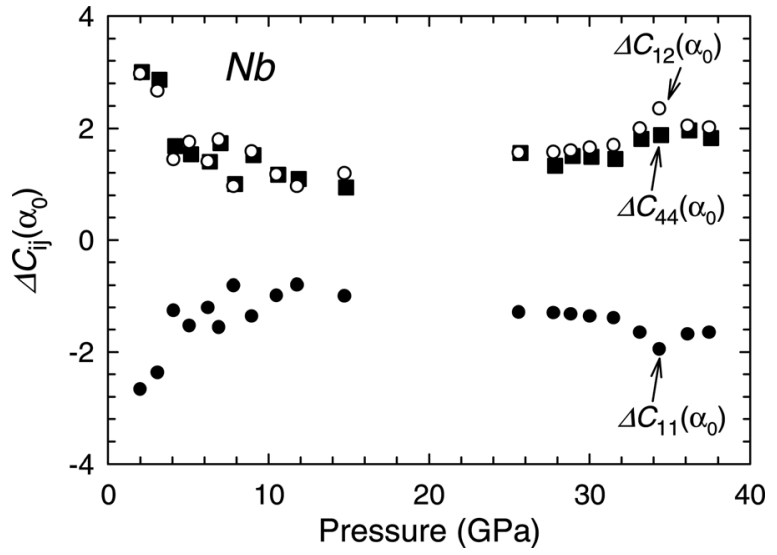

FIG. 12. The percentage deviations of $\left(C_{i j}\right)_{m}\left(\alpha_{0}\right)$ from the extrapolated $C_{i j}$ for different pressure runs, where $\left(C_{i j}\right)_{m}\left(\alpha_{0}\right)$ is the elastic moduli derived from the diffraction data with $\alpha=\alpha_{0}$.

\section{F. Plastic flow}

The sample undergoes severe plastic deformation upon nonhydrostatic compression in a high-pressure setup. Weidner et al. ${ }^{35}$ pointed out the importance of considering the plasticity of the solid sample along with the elastic deformation in the interpretation of the lattice strains. Attempts have been made more recently to include the effect of plastic deformation through numerical modeling. ${ }^{36,37}$ Karato stated that some important physics is missing in the model of Singh $^{12}$ and proposed a general theory. ${ }^{38}$ The Karato model postulates that each crystallite in a polycrystalline aggregate under nonhydrostatic compression experiences a stress that deforms the grain. Any attempt to compute the stress from the x-ray measured strains (elastic) and elastic moduli gives only the microscopic stress (the stress in crystallites). Extraction of the macroscopic stress (the stress in the bulk sample) requires the knowledge of the relationship between the two types of stresses. This is achieved in the Karato theory by including viscosity tensor along with the elastic compliance tensor. It may be noted that there exist some similarities between the Karato model and the earlier model (discussed in detail in $\mathrm{ref}^{15}$ ) that was used in the derivation of the lattice

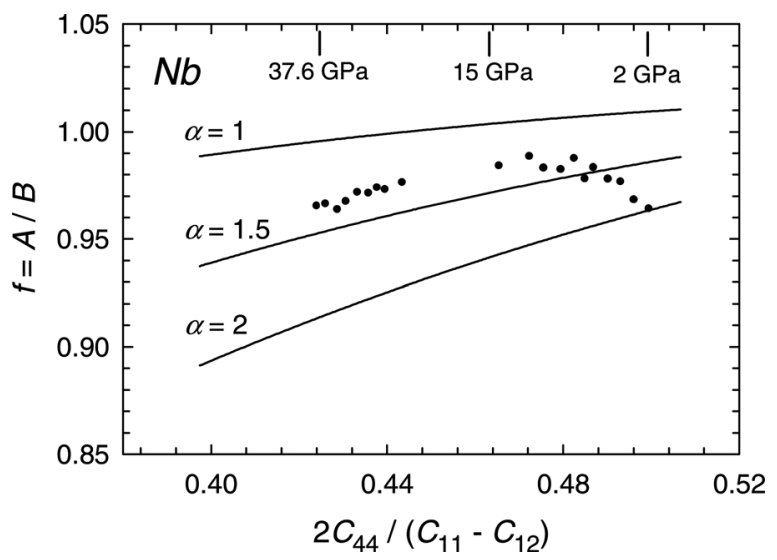

FIG. 13. The $f$ vs $x$ (Eqs. 6b-6e) computed with $\alpha$-values of $1,1.5$, and 2 (continuous lines). Here $x=2 C_{44}\left(C_{11}-C_{12}\right)$. The dots show the data computed with $\alpha=\alpha_{0}$ and $x$ corresponding to the pressures of the experimental runs. The pressures corresponding to a few $x$ are marked as a guide. strain equations. ${ }^{12}$ The lattice strain equations were developed specifically to explain the nonhydrostatic compression effects in the x-ray diffraction data taken with the opposed anvil setup at ambient temperature. In these experiments, the $\mathrm{x}$-ray diffraction patterns are recorded when the plastic flow accompanying the initial compression has ceased. In such cases, temporal changes in stresses and strain in the sample are absent. No attempt was, therefore, made to introduce any physical process that would account for time-dependent stresses and strains. This is a typical example of static stresses and strains. Only effect of plastic deformation of the sample is to establish stress gradients. The nature of flow due to the plastic deformation of the sample-gasket assembly in an opposed-anvil device is distinct from the flow of the sample in the relatively larger-volume devices ${ }^{31,32}$ and more so in presence of high-temperature creep. In case of the former, a state of static stress-strain is attained in a reasonably short time whereas, in case of the latter, the time-dependent stressstrain continues for long periods of time. The lattice strain equations derived earlier ${ }^{12-15}$ are not ideally suited for rheological studies of solids at high temperature. Still the use of lattice strain equations have resulted in satisfactory results. $^{31,32}$

\section{SUMMARY}

The compressive strength $t$ of niobium initially increases with pressure and exhibits a shallow maximum at $\sim 5 \mathrm{GPa}$ and a minimum at $\sim 12 \mathrm{GPa}$ followed by a monotonic increase at higher pressure. Niobium exhibits large elastic anisotropy at ambient pressure that increases with increasing pressure. Because of this, niobium exhibits large nonhydrostatic-compression effects in the diffraction data even though the $t$-values are low. The aggregate shear moduli of niobium computed from single-crystal elastic moduli by Voigt-ReussHill, Hashin-Shtrickman, and Kröner methods agree well. The Voigt shear modulus and diffraction shear modulus computed under Reuss limit do not constitute the upper and lower bounds, respectively, of the diffraction shear modulus even under small deformation. However, this does not constitute a violation of Hill theorem that applies to the aggregate shear modulus. For a good match between the set of $C_{i j}$ derived from the diffraction data and those obtained by the extrapolation of ambient pressure elasticity data, $\alpha>1$ is required. The $\alpha$-value required for this match decreases with increasing pressure. The assumption that the factor $f \cong 1$, which is made in the evaluation of $t$, is shown to be valid for niobium.

\section{ACKNOWLEDGMENTS}

A. K. S. thanks Director, NAL, for supporting this activity and M. Sujata and S. K. Bhaumik for helpful discussions. The authors thank S. Merkel for providing the software Multifit 4.2 and initial instruction for its use. Experiments were preformed at HPCAT (Sector 16), Advanced Photon Source (APS), Argonne National Laboratory. HPCAT is supported by CIW, CDAC, UNLV, and LLNL through funding from DOE-NNSA, DOE-BES, 
and NSF. APS is supported by DOE-BES, under Contract No. DE-AC02-06CH11357.

${ }^{1}$ L.-C. Ming and M. H. Manghnani, J. Appl. Phys. 49, 208 (1978).

${ }^{2}$ A. Fukizawa and Y. Fukai, J. Phys. Soc. Japan. 52, 2102 (1983).

${ }^{3}$ Y. Akahama, M. Kobayashi, and H. Kawamura, J. Phys. Soc. Jpn. 60, 3211 (1991).

${ }^{4} \mathrm{~K}$. Takemura, in Science and Technology of High Pressure, Proceedings of AIRAPT-17, Hawaii, edited by M. H. Manghnani, W. J. Nellis, and M.

F. Nicol (Universities Press, Hyderabad, India, 2000), p. 443 (1999).

${ }^{5}$ K. Takemura and A. K. Singh, Phys. Rev. B 73, 224119 (2006).

${ }^{6}$ R. G. McQueen, S. P. Marsh, J. W. Taylor, J. N. Fritz, and W. J. Carter, in High Velocity Impact Phenomena, edited by R. Kinslow (Academic Press, New York, 1970), p. 293.

${ }^{7}$ R. Ahuja, P. Söderlind, J. Trygg, J. Melsen, J. M. Wills, B. Johansson, and O. Eriksson, Phys. Rev. B 50, R14690 (1994).

${ }^{8}$ V. V. Struzhkin, Y. A. Timofeev, R. J. Hemley, and Ho-kwang Mao, Phys. Rev. Lett. 79, 4262 (1997).

${ }^{9}$ I. M. Lifshits, Sov. Phys. JETP 11, 1130 (1960).

${ }^{10}$ A. K. Singh and K. Takemura, J. Appl. Phys. 90, 3269 (2001); Erratum: 91, 5507 (2002).

${ }^{11}$ K. W. Katahara, M. H. Manghanani, and E. S. Fisher, J. Phys. F: Metal Phys. 9, 773 (1979)

${ }^{12}$ A. K. Singh, J. Appl. Phys. 73, 4278 (1993); Erratum: 74, 5920 (1993).

${ }^{13}$ A. K. Singh, H. K. Mao, J. Shu, and R. J. Hemley, Phys. Rev. Lett. 80, 2157 (1998).

${ }^{14}$ A. K. Singh, C. Balasingh, H. K. Mao, R. J. Hemley, and J. Shu, J. Appl. Phys. 83, 7567 (1998).

${ }^{15}$ A. K. Singh, J. Phys. Chem. Solids 65, 1589 (2004).

${ }^{16}$ A. K. Singh, J. Appl. Phys. 106, 043514 (2009).
${ }^{17}$ S. Merkel and T. Yagi, J. Appl. Phys. 76, 046109 (2005).

${ }^{18}$ W. Voigt, Lehrbuch der Kristallphysik (Teubner, Leipzig, 1928).

${ }^{19}$ E. Kröner, Z. Phys. 151, 504 (1958).

${ }^{20}$ R. de Wit, J. Appl. Cryst. 30, 510 (1997).

${ }^{21}$ F. Birch, J. Geophys. Res. 83, No. B3, 1257 (1978).

${ }^{22}$ R. Hill, Proc. Phys. Soc. (London), A 65, 349 (1952).

${ }^{23}$ A. Reuss and Z. Angew. Math. Mech. 9, 49 (1929).

${ }^{24}$ G. R. Barsch, Phys. Stat. Sol. 19, 129 (1967).

${ }^{25}$ A. K. Singh, in Science and Technology of High Pressure, Proceedings of AIRAPT-17, edited by M. H. Manghanani, W. J. Nellis, and M. F. Nicol (Universities Press, Hyderabad, India, 2000), pp. 62-67.

${ }^{26}$ S. Merkel, J. Phys.: Condens. Matter, 18, S949 (2006).

${ }^{27}$ Z. Hashin and S. Shtrikman, J. Mech. Phys. Solids 10, 335 (1962).

${ }^{28}$ Z. Hashin and S. Shtrikman, J. Mech. Phys. Solids 10, 343 (1962).

${ }^{29}$ L. I. Van Torne and G. Thomas, Acta Metall. 11, 881 (1963).

${ }^{30}$ G. E. Dieter, Mechanical Metallurgy - SI Metric Edition, adapted by D. Bacon (McGraw-Hill, Singapore, 1988), pp. 520-522.

${ }^{31}$ J. Chen, L. Li, T. Yu, H. Long, L. Wang, D. Weidner, and M. Vaughan, J. Phys.: Condens. Matter 18, S1049 (2006).

${ }^{32}$ J. Chen, L. Li, D. Weidner, and M. Vaughan, Phys. Earth Planet. Inter. 143-144, 347 (2004).

${ }^{33}$ T. S. Duffy, G. Shen, J. Shu, H.-K. Mao, R. J. Hemley, and A. K. Singh, J. Appl. Phys. 86, 6729 (1999).

${ }^{34}$ T. S. Duffy, G. Shen, D. L. Heinz, J. Shu, Y. Ma, H.-K. Mao, R. J. Hemley, A. K. Singh, Phys. Rev. B 60, 15063 (1999-II).

${ }^{35}$ D. J. Weidner, L. Li, M. Davis, and J. Chen, Geophys. Res. Lett. 31, L06621 (2004).

${ }^{36}$ P. C. Burnley and D. Zhang, J. Phys.: Condens. Matter 20, 28201 (2008).

${ }^{37}$ S. Merkel, C. Tomé, and H.-R. Wenk, Phys. Rev. B 79, 064110 (2009).

${ }^{38}$ S.-i. Karato, Phys. Rev. B 79, 214106 (2009). 